\title{
Surface energy and work function of elemental metals
}

\section{Skriver, Hans Lomholt; Rosengaard, N. M.}

\section{Published in:}

Physical Review B

Link to article, DOI:

10.1103/PhysRevB.46.7157

Publication date:

1992

\section{Document Version}

Publisher's PDF, also known as Version of record

Link back to DTU Orbit

Citation (APA):

Skriver, H. L., \& Rosengaard, N. M. (1992). Surface energy and work function of elemental metals. Physical Review B, 46(11), 7157-7168. https://doi.org/10.1103/PhysRevB.46.7157

\section{General rights}

Copyright and moral rights for the publications made accessible in the public portal are retained by the authors and/or other copyright owners and it is a condition of accessing publications that users recognise and abide by the legal requirements associated with these rights.

- Users may download and print one copy of any publication from the public portal for the purpose of private study or research.

- You may not further distribute the material or use it for any profit-making activity or commercial gain

- You may freely distribute the URL identifying the publication in the public portal

If you believe that this document breaches copyright please contact us providing details, and we will remove access to the work immediately and investigate your claim 


\title{
Surface energy and work function of elemental metals
}

\author{
H. L. Skriver and N. M. Rosengaard \\ Laboratory of Applied Physics, Technical University of Denmark, DK-2800 Lyngby, Denmark
}

(Received 22 May 1992)

\begin{abstract}
We have performed an $a b$ initio study of the surface energy and the work function for six closepacked surfaces of 40 elemental metals by means of a Green's-function technique, based on the linear-muffin-tin-orbitals method within the tight-binding and atomic-sphere approximations. The results are in excellent agreement with a recent full-potential, all-electron, slab-supercell calculation of surface energies and work functions for the $4 d$ metals. The present calculations explain the trend exhibited by the surface energies of the alkali, alkaline earth, divalent rare-earth, $3 d, 4 d$, and $5 d$ transition and noble metals, as derived from the surface tension of liquid metals. In addition, they give work functions which agree with the limited experimental data obtained from single crystals to within $15 \%$, and explain the smooth behavior of the experimental work functions of polycrystalline samples as a function of atomic number. It is argued that the surface energies and work functions calculated by present day $a b$ initio methods are at least as accurate as the experimental values.
\end{abstract}

\section{INTRODUCTION}

The surface energy and the work function are the two most fundamental electronic properties of a metallic surface, and their determination is of great importance in the understanding of a wide range of surface phenomena. This includes growth rate, the form of crystallites, sintering, catalytic behavior, adsorption, surface segregation, and the formation of grain boundaries. In spite of considerable experimental efforts, it appears that for many metals the recommended values of both quantities have uncertainties of unknown magnitude. This is, for instance, true for the surface energies derived from the surface tension of liquid metals and listed by de Boer et al. ${ }^{1}$ Nonetheless, to our knowledge the compilation by these authors represents the most consistent choice of surface energies in existence. Similarly, the work functions listed by Michaelson ${ }^{2}$ are derived mainly from measurements on polycrystalline samples and in many cases have not been confirmed by ultrahigh-vacuum measurements. In this situation one may turn to theoretical models and in particular to $a b$ initio calculations as a guide, especially since the accuracy of such calculations has recently improved considerably.

In the last two decades there have been several theoretical studies of surface properties, starting with the pioneering jellium calculations of Lang and Kohn. ${ }^{3,4}$ These model calculations were later improved by Perdew and co-workers, ${ }^{5-7}$ and they explained the trends exhibited by the work function and the surface energy of the simple and noble metals. Nieminen and Hodges ${ }^{8}$ used band estimates of the Fermi level and the experimentally obtained work function to deduce the surface dipole barrier for transition metals. Weinert and Watson ${ }^{9}$ used a different approach in the form of overlapping atomic charge densities to estimate the work function of some 30 metals, including both simple and transition metals. Their results gave a good description of the experimentally ob- served trends but overestimated the work functions by, on the average, $80 \%$.

In the past, $a b$ initio calculations of surface properties have been performed only in particular cases, ${ }^{10-27}$ mainly because of the large computer resources required. However, recently Skriver and Rosengaard ${ }^{28}$ implemented an efficient self-consistent Green's-function technique $^{28}$ based on the linear muffin-tin-orbital (LMTO) method and used it to calculate the work function of alkali, alkaline earth, transition, and noble metals. ${ }^{29}$ At the same time Methfessel, Hennig, and Scheffler ${ }^{30}$ presented the first comprehensive study of surface relaxation, surface energy, and work function for the $4 d$ metals by means of their full-potential LMTO slab method. It is gratifying to see that the two calculations, which differ in both approach and level of approximation, give work functions and surface energies for the $4 d$ metals which typically agree to better than $10 \%$.

Since the preliminary account of the present work ${ }^{29}$ our Green's-function technique has been improved in several respects. Most notable is the use of linear-response theory and a linearized version of the Dyson equation to improve convergence. This cuts the number of iterations by an order of magnitude. Furthermore, in the bulk calculations we apply the second-order LMTO Hamiltonian also used in the Green's-function technique, we use the Brillouin zone appropriate to each particular surface, and obtain the kinetic energy by integration of the Green's function on a complex energy contour. As a result we obtain an effective cancellation of errors and improved accuracy in the calculated surface energies.

Here, we report a comprehensive series of calculations for the closed-packed surfaces of alkali, alkaline earth, divalent rare earth, $3 d, 4 d$, and $5 d$ transition and noble metals. The main objective of the study is to provide a consistent set of work functions and surface energies, which may serve as a database for the construction of models of a wider range of surface phenomena. 


\section{COMPUTATIONAL METHOD}

The tight-binding linear-muffin-tin-orbitals (TBLMTO) Green's-function technique as implemented by Skriver and Rosengaard ${ }^{28}$ is based on the work by Andersen and co-workers. ${ }^{31-37}$ An essential aspect of the technique is the ability, within the atomic sphere approximation (ASA) and in the tight-binding representation, to generate the Green's-function matrices for a real, twodimensional interface by a simple and efficient procedure. Since the details of the technique may be found in Ref. 28 we shall here restrict ourselves to an outline of the major steps, with special emphasis on the results of linearresponse theory.

\section{A. Surface Green's function}

The Hamiltonian Green's function matrix, $G(z)$, for the infinite crystal is most conveniently obtained from the LMTO-ASA equations

$$
\left(z \mathbf{1}-H^{\gamma}\right) G^{\gamma}(z)=1
$$

within the orthogonal representation. Here, $z$ is a complex energy and $H^{\gamma}$ the second-order LMTO Hamiltonian

$$
H^{\gamma}(\mathbf{k})=C+\sqrt{\Delta} S^{\gamma}(\mathbf{k}) \sqrt{\Delta}
$$

evaluated at a particular vector $\mathbf{k}$ in reciprocal space. The center $C$, bandwidth $\Delta$, and $\gamma$ potential parameters which enter the Hamiltonian are taken from a selfconsistent bulk calculation while the structure constants $S^{\gamma}$ are obtained by matrix inversion of the most localized structure constants $S^{\beta}$, evaluated by real-space summations once and for all for a given surface.

In a layer representation the Green's-function matrix may be expressed as

$$
\begin{aligned}
G_{R_{\perp}^{\prime} L^{\prime}, R_{\perp} L}^{\gamma}\left(\mathbf{k}_{\|}, z\right)= & \frac{d_{\perp}}{2 \pi} e^{-i \mathbf{k}_{\|} \cdot\left(\mathbf{R}_{\|}-\mathbf{R}_{\|}^{\prime}\right)} \\
& \times \int_{0}^{2 \pi / d_{\perp}} d k_{\perp} e^{-i k_{\perp}\left(R_{\perp}-R_{\perp}^{\prime}\right)} \\
& \times G_{R^{\prime} L^{\prime}, R L}^{\gamma}(\mathbf{k}, z),
\end{aligned}
$$

where $L$ refers to the combined angular-momentum quantum numbers $(l, m), d_{\perp}$ is a layer distance, $\mathbf{k}=\left(\mathbf{k}_{\|}, k_{\perp}\right)$ is the decomposed reciprocal-space vector, and $\mathbf{R}=$ $\left(\mathbf{R}_{\|}, R_{\perp}\right)$ denotes sites in the three-dimensional (3D) cell.

The Green's-function matrices for a semi-infinite crystal are obtained by a Löwdin downfolding technique ${ }^{38}$ which hinges on the fact that the LMTO structure constants in the most localized representation are short ranged, i.e.,

$$
S_{A B}^{\beta}=0 \text { for }\{A B\} \neq\{a b\} .
$$

Here, $A B$ refers to the two half-spaces separated by the surface and $\{a b\}$ is the small subset of layers where the hopping is nonzero. After the transformation ${ }^{28,34,36}$ of the layer Green's-function matrix (3) into the form defined by the Korringa-Kohn-Rostoker (KKR) ASA equa- tions in the $\beta$ representation the Löwdin downfolded $i d e a l$ Green's-function matrices for the bulk $(X)$ and the vacuum $(V)$ are given by

$$
\begin{aligned}
& \tilde{g}_{A A}^{X}=g_{A A}^{X}-g_{A b}^{X}\left(g_{b b}^{X}\right)^{-1} g_{b A}^{X}, \\
& \tilde{g}_{B B}^{V}=g_{B B}^{V}-g_{B a}^{V}\left(g_{a a}^{V}\right)^{-1} g_{a B}^{V} .
\end{aligned}
$$

As explained in Ref. 28 the vacuum Green's-function matrices are found directly from an analytic expression for the LMTO structure constants and no $\mathbf{k}_{\perp}$ integration is needed.

The surface Green's-function matrices $g$ for the unrelaxed, combined system is found from the definition of the KKR-ASA Green's function written in the block form

$$
\left(\begin{array}{cc}
\left(\tilde{g}_{a a}^{X}\right)^{-1} & -S_{a b} \\
-S_{b a} & \left(\tilde{g}_{b b}^{V}\right)^{-1}
\end{array}\right)\left(\begin{array}{cc}
g_{a a} & g_{a b} \\
g_{b a} & g_{b b}
\end{array}\right)=\left(\begin{array}{ll}
1_{a a} & 0_{a b} \\
0_{a b} & 1_{b b}
\end{array}\right) .
$$

Finally, the effect of relaxing the potentials close to the surface is found from the finite Dyson equation

$$
g^{s}=g+g \Delta P^{s} g^{s},
$$

where the surface Green's-function matrix $g^{s}$ is expressed in terms of the unrelaxed Green's-function matrix and the diagonal potential-function matrix $\Delta P^{s}$ which describes the relaxation of the potentials.

\section{B. Linear-response theory}

In the iterations towards self-consistency, there are two problems which slow down the convergence. The first is the time-consuming numerical solution of the Dyson equation (8) which must be performed at each iteration. The second is the well-known instability caused by the long-range Coulomb interactions, leading to oscillations in the potential as the iterations progress. Traditionally, the latter problem is solved by mixing input and output potentials or charge densities from previous iterations to form the input for the next iteration. Such mixing schemes correspond to a linear solution of a general nonlinear problem which may not be appropriate in all cases.

In a recent study of the band offsets in heterojunctions Lambrecht, Segall, and Andersen ${ }^{39}$ showed that linearresponse theory gave an accurate estimate of the size of the electrostatic dipole at the interface. Combined with the Green's-function technique, their result means that one may use linear-response theory, based on the correct nonlinear problem, to estimate the input potential for the next iteration. As a result, the mixing of the charges causing the most troublesome part of the oscillatory behavior will be optimized to the surface situation. If, in addition, one solves a linearized version of the Dyson equation, one needs only to solve the complete Dyson equation when a self-consistent linear solution has been obtained, thus overcoming simultaneously the first problem.

Let us assume that we have solved the Dyson equation (8) for a specified input potential. Hence, we know the 
charge density in our system, and in particular we know the net charge $Q_{R}$ in the atomic spheres through the surface region. If we then shift the potential in each sphere by a small amount $\delta v_{R^{\prime}}$, the change in the charges $\delta Q_{R}$ may be found to linear order from

$$
\delta Q_{R}=\sum_{R^{\prime}} \chi_{R, R^{\prime}} \delta v_{R^{\prime}}
$$

where $\chi_{R, R^{\prime}}$ is the linear-response matrix given below. If we impose a shift $\delta H^{\gamma}$ on the LMTO Hamiltonian (2) the corresponding change in the Green's function may be found from the linear Dyson equation

$$
\delta G^{\gamma}=G^{\gamma} \delta H^{\gamma} G^{\gamma}
$$

as obtained by keeping terms to linear order in (1). If we neglect the small change in the bandwidth parameter $\Delta$ caused by $\delta v_{R^{\prime}}$ we have that

$$
\delta H_{R^{\prime}}^{\gamma}=\delta C_{R^{\prime}}=\delta v_{R^{\prime}}
$$

and hence the linear-response matrix may be obtained from the Green's-function matrices as the integral

$$
\begin{array}{r}
\chi_{R R^{\prime}}=\frac{1}{2 \pi i} \oint d z \int d \mathbf{k}_{\|} \sum_{L L^{\prime}} G_{R L, R^{\prime} L^{\prime}}^{\gamma}\left(\mathbf{k}_{\|}, z\right) \\
\times G_{R^{\prime} L^{\prime}, R L}^{\gamma}\left(\mathbf{k}_{\|}, z\right),
\end{array}
$$

which is easily evaluated from the known Green'sfunction matrices.

Given the change in the net charges calculated by linear-response theory (9) the change in the long-range Coulomb potential is obtained as the Madelung sum

$$
\delta v_{R}=\frac{1}{S} \sum_{R^{\prime}} M_{R, R^{\prime}}^{s s} \delta Q_{R^{\prime}},
$$

where $S$ is the average Wigner-Seitz radius and $\mathbf{M}_{R, R^{\prime}}^{s s}$ the two-dimensional Madelung matrix given in Eq. (59) of Ref. 28. Thereby the change to linear order in the output potential upon a small disturbance of the input is given by

$$
\delta v_{\text {out }}=\mathbf{A} \delta v_{\text {in }}
$$

where we have defined a new matrix

$$
\mathbf{A}=\mathbf{M} \cdot \chi
$$

as the product of the linear-response matrix and the twodimensional Madelung matrix.

Self-consistency requires that the input and output potentials at a given iteration $(n+1)$ are equal, i.e.,

$$
v_{\text {in }}^{(n)}+\delta v_{\text {in }}^{(n+1)}=v_{\text {out }}^{(n)}+\mathbf{A} \delta v_{\text {in }}^{(n+1)} \text {. }
$$

This equation may be solved to give the change in the input potential in the form

$$
\delta v_{\text {in }}^{(n+1)}=(1-\mathbf{A})^{-1}\left(v_{\text {out }}^{(n)}-v_{\text {in }}^{(n)}\right),
$$

where the matrix to be inverted numerically only has the dimension of the number of layers in the surface region. Thus, at each iteration and to linear order, the long-range part of the input potential for the following iteration is simply given by

$$
v_{\mathrm{in}}^{(n+1)}=v_{\mathrm{in}}^{(n)}+\delta v_{\mathrm{in}}^{(n+1)}
$$

This solution does not automatically lead to chargeneutrality but one may impose a small $R$-independent potential shift $\delta V$ given by

$$
\delta V=-\sum_{R R^{\prime}} \chi_{R R^{\prime}} \delta v_{\mathrm{in} ; R^{\prime}}^{(n+1)} / \sum_{R R^{\prime}} \chi_{R R^{\prime}}
$$

which will guarantee that the entire two-dimensional unit cell contains no net charge. The complete long-range part of the input potential corresponding to a neutral distribution of net charges may therefore be found as

$$
v_{\mathrm{in} ; R}^{(n+1)}=v_{\mathrm{in} ; R}^{(n)}+\delta v_{\mathrm{in} ; R}^{(n+1)}+(1-\mathbf{A})^{-1} \delta V .
$$

Viewed as a function of $R$, this potential is a stepwise approximation to the surface dipole barrier, and is used as the boundary condition on the numerical solution to the Poisson equation inside the individual atomic spheres.

\section{Details of the calculations}

At the outset of a surface calculation, one needs starting potentials as well as total energies corresponding to the perfect, infinite crystal of the atomic species that form the surface. To obtain this input, we perform selfconsistent bulk calculations by means of the second-order Hamiltonian (2) also used in the Green's-function technique, and calculate the kinetic energy by integrating the Green's function on a complex energy contour. Also, although it is more time consuming, we use the Brillouin zone appropriate to each particular surface and perform bulk calculations for each surface facet. As a result we obtain an effective cancellation of errors and improved accuracy in the calculated surface energies.

Based on convergence tests we use a surface region consisting of four layers of metal plus two layers of empty spheres simulating the vacuum. For the $k$-space integration we use 36 special points ${ }^{40}$ in the irreducible part of the two-dimensional Brillouin zone for the fcc (100) and bcc (100) surfaces, 45 points for the fcc (111) and hcp (001) surfaces, and 64 points for the fcc (110) and bcc (110) surfaces. Finally, we calculate the moments of the state density by means of a Gaussian integration technique with 16 points distributed exponentially on a semicircle in the complex plane to emphasize the contributions close to the Fermi level. In a few difficult cases with surface states close to the Fermi level we include a Fermi function corresponding to $300 \mathrm{~K}$ in the manner described by Zeller et al. ${ }^{41}$ As a result, we expect the calculated work functions and surface energies to be converged to within $0.1 \mathrm{eV}$ and $5 \mathrm{~mJ} / \mathrm{m}^{2}$, respectively.

As pointed out by Aldén et al. ${ }^{42}$ the work function depends critically upon the exchange-correlation functional, since electrons are removed from a region of space with metallic density to a region of zero density. Hence, any constant difference in the exchange-correlation potential is directly reflected in the calculated work functions. In the calculations for the alkali metals ${ }^{28}$ we 
found that the functional of Ceperley and Alder ${ }^{43}$ in the form parametrized by Perdew and Zunger ${ }^{44}$ gave the best overall agreement with the measured work functions. We have therefore used this functional throughout the present work.

The one-electron potentials are calculated within the ASA, i.e., they are spherically symmetric inside overlapping spheres. In particular, we note that the exchangecorrelation potential is obtained from the spherically averaged electron density and not from the full density. Test calculations show that this approximation tends to overestimate the work functions by typically $0.2 \mathrm{eV}$. The electrostatic potential is also obtained within the ASA, but in addition to the monopole Madelung contribution from the net charges, it contains a contribution from the second, i.e., dipole terms in the multipole expansion of the charge density in the other spheres. Hence, in the present implementation the surface dipole barrier has two contributions, one from the monopoles, i.e., the net charges in the spheres and one from the dipole charges in the spheres.

The surface energy is the work required to form a unit area of surface, and is the solid analog of the surface tension of a liquid. In the present case and within the BornOppenheimer, local-density, atomic sphere, and frozencore approximations it is simply calculated as the difference

$$
\gamma=E_{2 \mathrm{D}}(N)-N E_{3 \mathrm{D}}
$$

between the total energy of the $N$ atoms in the surface region and $N$ times the total energy of single atoms in the bulk.

Within the ASA, and for a bulk cell with only equivalent atoms, the zero of energy is taken to coincide with the electrostatic potential at the surface of the (neutral) atomic sphere, and the work function $W$ of the corresponding semi-infinite metal may then be expressed as the difference

$$
W=\phi-E_{F},
$$

between the electrostatic surface dipole $\phi$ and the Fermi level $E_{F}$ for the bulk crystal on the ASA energy scale. The inclusion of the above-mentioned dipole-charge contribution to the electrostatic potential reduces the surface dipole barrier by up to $2 \mathrm{eV}$, and hence is essential for the accuracy of the calculated work function.

\section{RESULTS}

In the following we shall present surface energies and work functions for 40 metallic elements, as obtained by the procedure described in Sec. II. All calculations are performed at the experimentally observed equilibrium volumes, and surface relaxations are neglected. For each metal, the surface energy and the work function have been calculated for the most close-packed surface of the experimentally observed crystal structure. These results are listed in the first line for a given metal in Tables I-IV and together with results for other close-packed surfaces also listed in the tables they will be compared with the two compilations of experimental data $a^{1,2}$ and with recent jellium ${ }^{7}$ and $a b$ initio calculations. ${ }^{30}$

\section{A. Nontransition metals}

Traditionally, the surface properties of the so-called simple metals have been calculated within the jellium model, to which was added the effect of the ion lattice treated by perturbation theor $y^{3,4}$ or by a variational approach. ${ }^{7}$ In Table I we therefore compare our calculated surface energies and work functions for the alkali, alkaline earth, divalent rare earth, and aluminum with the most recent jellium-type results.

\section{Surface energy}

From Table I it is seen that the surface energies for the heavy alkali metals, $\mathrm{K}-\mathrm{Cs}$, obtained by the present and jellium-based methods agree to within $7 \%$. In contrast to this, the deviations for the two light alkali metals, $\mathrm{Li}$ and $\mathrm{Na}$, are $40 \%$ and $60 \%$, respectively. For the divalent metals, $\mathrm{Mg}-\mathrm{Ba}$, the deviations vary from $15 \%$ to $8 \%$. It is furthermore seen that the present surface energies in all cases are higher than the corresponding jellium-based values, and in closer agreement with the surface energies derived from the surface tension of liquid metals.

One of the justifications for what has become known as the Miedema model of cohesion has been the linear relationship between the surface energy and the interatomic electron density, $n_{\mathrm{WS}}{ }^{45}$ In Fig. 1 we have therefore fitted the calculated surface energies to $r_{s}^{-3} \sim n_{\mathrm{WS}}$. It is interesting to note that on this scale, which is slightly different from the one used by de Boer et al., ${ }^{1}$ the calculated surface energies are closer to the linear relationship than are the experimental values.

We take the agreement between the calculated surface energies and the values derived by de Boer et al. ${ }^{1}$ demonstrated in Fig. 1 to mean that local-density theory and the present Green's-function technique can provide surface energies which are at least as accurate as those derived from experiments.

\section{Work function}

From Table I it is seen that the work functions of the alkali metals, $\mathrm{Li}-\mathrm{Cs}$, obtained by the two methods agree to within $8 \%$. For the divalent metals, $\mathrm{Mg}-\mathrm{Ba}$, the deviations are larger, and in $\mathrm{Ba}$ the present work function is $19 \%$ lower than the jellium result. This is, however, quite understandable, since the jellium-type model is expected to be less accurate for divalent metals than for the simple, monovalent metals.

In the comparison with experiment, Fig. 2, we find that the calculated work functions in most cases are larger than the measured values. This is in agreement with the fact that the calculations are performed for single, close-packed surfaces, while the experimental values are obtained from measurements on polycrystalline samples and reflect an average over different surfaces facets, some 
TABLE I. Work functions and surface energies for monovalent, divalent, and trivalent nontransition metals. The experimental work functions in parentheses are polycrystalline values.

\begin{tabular}{|c|c|c|c|c|c|c|c|c|}
\hline \multirow[t]{3}{*}{ Metal } & \multirow[t]{3}{*}{ Surface } & \multicolumn{3}{|c|}{ Work function } & \multicolumn{4}{|c|}{ Surface energy } \\
\hline & & Jellium $^{\mathrm{a}}$ & Present & Expt. ${ }^{b}$ & Jellium $^{a}$ & & & Expt. ${ }^{c}$ \\
\hline & & $(\mathrm{eV})$ & $(\mathrm{eV})$ & $(\mathrm{eV})$ & $\left(\mathrm{J} / \mathrm{m}^{2}\right)$ & $(\mathrm{eV})$ & $\left(\mathrm{J} / \mathrm{m}^{2}\right)$ & $\left(\mathrm{J} / \mathrm{m}^{2}\right)$ \\
\hline $\mathrm{Li}$ & $\begin{array}{l}\text { bcc }(110) \\
\text { bcc }(100)\end{array}$ & $\begin{array}{l}3.09 \\
2.92\end{array}$ & $\begin{array}{l}3.33 \\
3.15\end{array}$ & $(2.9)$ & $\begin{array}{l}0.326 \\
0.371\end{array}$ & $\begin{array}{l}0.247 \\
0.332\end{array}$ & $\begin{array}{l}0.458 \\
0.436\end{array}$ & 0.525 \\
\hline $\mathrm{Na}$ & $\begin{array}{l}\text { bcc }(110) \\
\text { bcc }(100)\end{array}$ & $\begin{array}{l}2.75 \\
2.58\end{array}$ & $\begin{array}{l}2.94 \\
2.76\end{array}$ & $(2.75)$ & $\begin{array}{l}0.190 \\
0.216\end{array}$ & $\begin{array}{l}0.242 \\
0.263\end{array}$ & $\begin{array}{l}0.307 \\
0.236\end{array}$ & 0.260 \\
\hline $\mathrm{K}$ & $\begin{array}{l}\text { bcc }(110) \\
\text { bcc }(100) \\
\text { fcc }(111)\end{array}$ & $\begin{array}{l}2.37 \\
2.21\end{array}$ & $\begin{array}{l}2.38 \\
2.34 \\
2.41\end{array}$ & $(2.30)$ & $\begin{array}{l}0.111 \\
0.115\end{array}$ & $\begin{array}{l}0.139 \\
0.220 \\
0.132\end{array}$ & $\begin{array}{l}0.116 \\
0.129 \\
0.112\end{array}$ & 0.130 \\
\hline $\mathrm{Rb}$ & $\begin{array}{l}\text { bcc }(110) \\
\text { bcc }(100) \\
\text { fcc }(111)\end{array}$ & $\begin{array}{l}2.28 \\
2.12\end{array}$ & $\begin{array}{l}2.32 \\
2.22 \\
2.29 \\
\end{array}$ & $(2.16)$ & $\begin{array}{l}0.086 \\
0.098\end{array}$ & $\begin{array}{l}0.127 \\
0.207 \\
0.120\end{array}$ & $\begin{array}{l}0.092 \\
0.107 \\
0.089\end{array}$ & 0.110 \\
\hline Cs & $\begin{array}{l}\text { bcc }(110) \\
\text { bcc }(100) \\
\text { fcc }(111)\end{array}$ & $\begin{array}{l}2.17 \\
2.01\end{array}$ & $\begin{array}{l}2.09 \\
2.03 \\
2.10 \\
\end{array}$ & $(2.14)$ & $\begin{array}{l}0.069 \\
0.079\end{array}$ & $\begin{array}{l}0.117 \\
0.210 \\
0.111\end{array}$ & $\begin{array}{l}0.072 \\
0.092 \\
0.070 \\
\end{array}$ & 0.095 \\
\hline $\mathrm{Be}$ & hcp (001) & & 5.62 & $(4.98)$ & & 0.583 & 2.122 & 2.70 \\
\hline $\mathrm{Mg}$ & hcp (001) & 3.44 & 3.86 & $(3.66)$ & 0.554 & 0.356 & 0.642 & 0.76 \\
\hline $\mathrm{Ca}$ & $\begin{array}{l}\text { fcc }(111) \\
\text { bcc }(110)\end{array}$ & 3.10 & $\begin{array}{l}2.86 \\
2.84 \\
\end{array}$ & $(2.87)$ & 0.325 & $\begin{array}{l}0.296 \\
0.293\end{array}$ & $\begin{array}{l}0.352 \\
0.339\end{array}$ & 0.49 \\
\hline $\mathrm{Sr}$ & $\begin{array}{l}\text { fcc }(111) \\
\text { bcc }(110)\end{array}$ & 2.94 & $\begin{array}{l}2.42 \\
2.39\end{array}$ & $(2.59)$ & 0.256 & $\begin{array}{l}0.287 \\
0.291 \\
\end{array}$ & $\begin{array}{l}0.287 \\
0.282\end{array}$ & 0.41 \\
\hline $\mathrm{Ba}$ & $\begin{array}{l}\text { bcc }(110) \\
\text { fcc }(111)\end{array}$ & 2.83 & $\begin{array}{l}2.28 \\
2.23 \\
\end{array}$ & $(2.7)$ & 0.233 & $\begin{array}{l}0.286 \\
0.277 \\
\end{array}$ & $\begin{array}{l}0.260 \\
0.258\end{array}$ & 0.37 \\
\hline $\mathrm{Ra}$ & bcc (110) & & 2.25 & & & 0.288 & 0.247 & \\
\hline $\mathrm{Eu}$ & bcc (110) & & 2.42 & $(2.5)$ & & 0.317 & 0.342 & 0.45 \\
\hline $\mathrm{Yb}$ & $\begin{array}{l}\text { fcc }(111) \\
\text { bcc }(110)\end{array}$ & & $\begin{array}{l}2.51 \\
2.45 \\
\end{array}$ & & & $\begin{array}{l}0.318 \\
0.328\end{array}$ & $\begin{array}{l}0.391 \\
0.391 \\
\end{array}$ & 0.50 \\
\hline $\mathrm{Al}$ & fcc $(111)$ & 3.72 & 4.54 & 4.24 & 0.921 & 0.56 & 1.27 & 1.16 \\
\hline
\end{tabular}

${ }^{\text {a }}$ See Ref. 7.

${ }^{\mathrm{b}}$ See Ref. 2 .

'see Ref. 1.

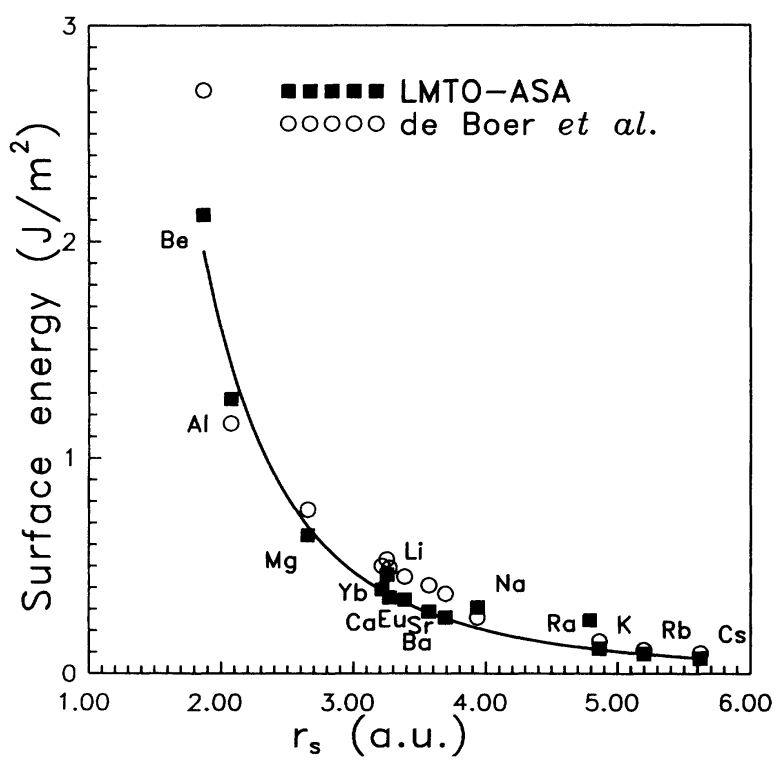

FIG. 1. The calculated surface energies for nontransition metals, solid squares, compared to the structure-independent values derived from the surface tension of liquid metals, open circles (Ref. 1). The solid line represents a fit of $r_{s}^{-3}$ to the calculated energies.

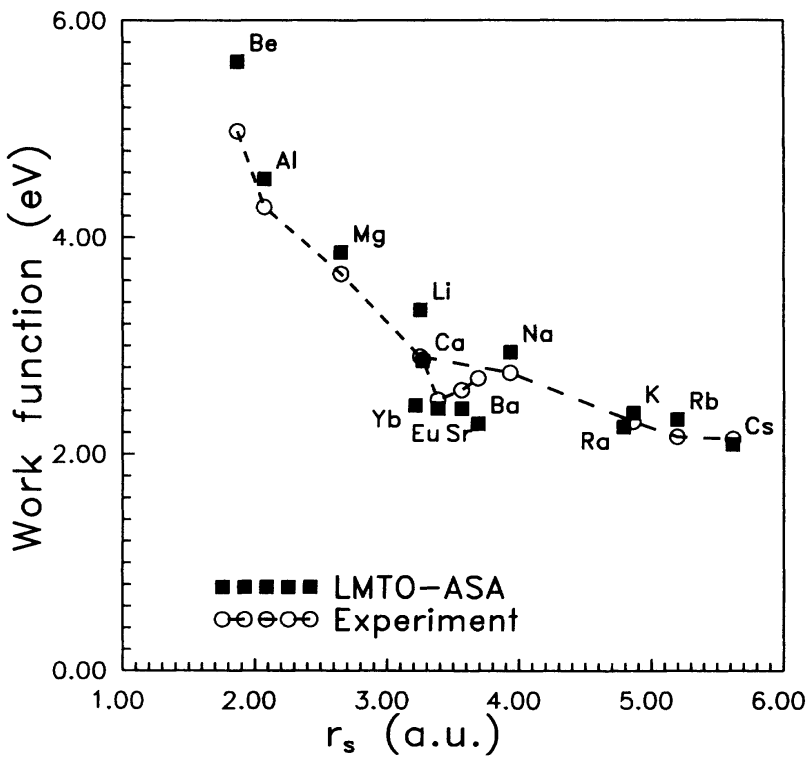

FIG. 2. The calculated work function for nontransition metals including the alkali, alkaline earth, divalent rare earths, and aluminum, solid squares, compared to the compilation of experimental polycystalline data by Michaelson (Ref. 2), open circles. The results are plotted as functions of the density parameter $r_{s}$, and the lines connecting the experimental values are guides to the eye. 
of which are relatively open and have low work functions. Hence, one would expect the calculated values to represent an upper bound on the experimental results, as indeed they do.

If one considers the work functions in Fig. 2 as functions of the density variable $r_{s}$, one finds that the values as a rule decrease with increasing $r_{s}$. In fact, the work functions of the monovalent metals lie close to a line from $\mathrm{Li}$ to $\mathrm{Cs}$ while those of the divalent metals lie close to a line from Be to Eu. This trend is followed by the calculated as well as the experimental values, except for the sequence $\mathrm{Ca}-\mathrm{Eu}-\mathrm{Sr}-\mathrm{Ba}$, where the measured values increase with $r_{s}$. The jellium-type results for Ca-Sr-Ba, Table I, support the decreasing trend, and hence one may argue that the deviation between theory and measurement in this sequence is caused by experimental difficulties.

On the ASA energy scale the work function of a metal surface is obtained as the difference between the electrostatic dipole barrier and the Fermi level. To understand the variation with atomic number we have therefore plotted the two terms of Eq. (22) for the alkali and alkalineearth metals in Fig. 3.

A simple estimate of the dipole barrier may be obtained within a two-plate condenser model. One finds

$$
\phi=M \frac{q}{S}
$$

where $q$ is the charge per atom in the first surface layer and $M$ is a constant of the order 10 depending on crystal structure and surface facet. The dependence on the Wigner-Seitz radius $S$ is a consequence of the fact that as the layer distance increases so does the surface area per atom. With $q$ independent of atomic number, which is approximately correct for both the alkali and the alkaline-

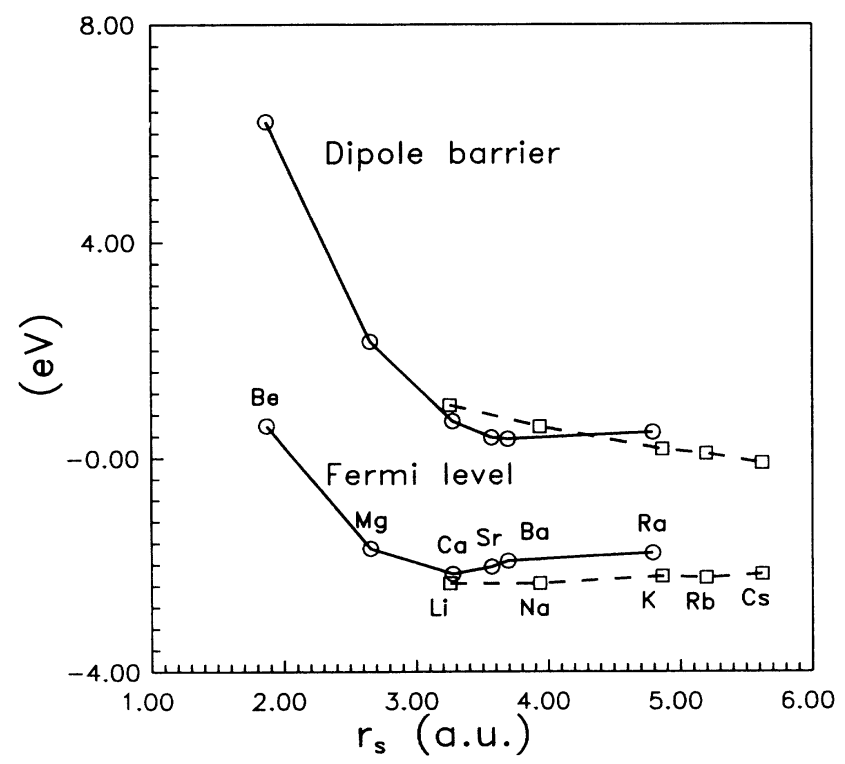

FIG. 3. The calculated dipole barrier and Fermi level for the alkali and alkaline-earth metals plotted as functions of the density parameter $r_{s}$. earth metals, the decrease in the dipole barrier with atomic number in Fig. 3 follows immediately. The deviation from the straight $1 / S$ dependence can be traced to a breakdown of the constant $q$ approximation and to the effect of the dipole-charge contribution not included in the simple condenser model.

The Fermi level in simple metals may be estimated from

$$
E_{F}=\left(\frac{9 \pi}{4}\right)^{1 / 3} \frac{1}{r_{s}^{2}}-\frac{2 Z_{\mathrm{WS}}}{S}
$$

where the first term on the right-hand side is the freeelectron Fermi level and the second an approximate exchange-correlation potential at the Wigner-Seitz radius, which provides a good estimate of the bottom of the free-electron band. With $Z_{\mathrm{WS}}=0.8$ the balance between kinetic and potential energy inherent in Eq. (24) explains the slow increase in the Fermi level of the alkali metals as a function of atomic number. For the high densities realized in $\mathrm{Be}$ and $\mathrm{Mg}$, i.e., $r_{s}<3$, the first term in Eq. (24) dominates and, as a result, the Fermi level exhibits a minimum in the alkaline-earth series.

\section{B. Transition metals}

$A b$ initio surface calculations for transition metals require large computer resources and have typically been performed only in particularly interesting cases. ${ }^{10-27} \mathrm{As}$ a result, the recent study of surface relaxation, surface energy, and work function for the $4 d$ metals by Methfessel, Hennig, and Scheffler ${ }^{30}$ is the first comprehensive study

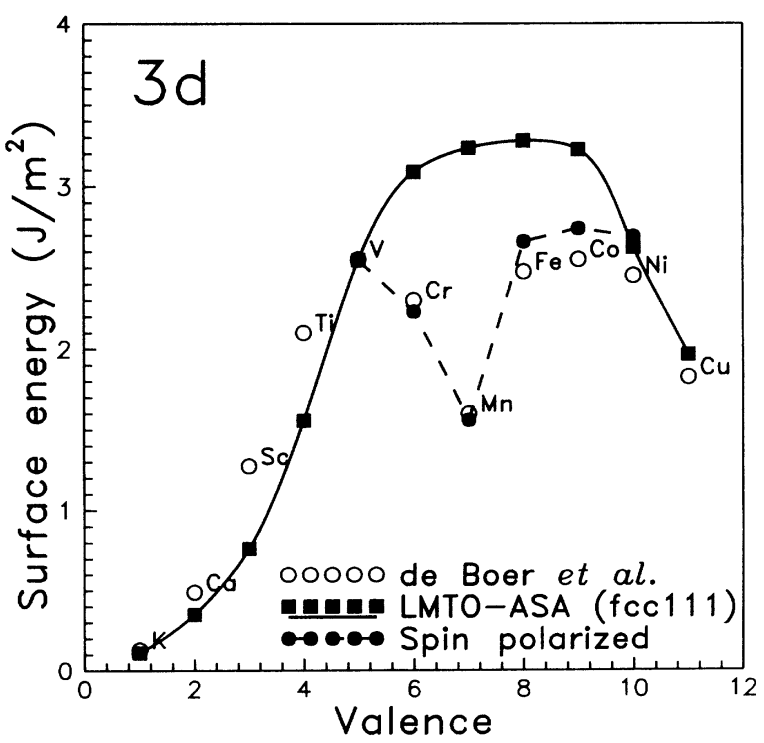

FIG. 4. The calculated surface energy for the fcc (111) surfaces of the $3 d$ metals, solid squares, compared to the structure-independent surface energies derived from the surface tension of the liquid metals, open circles (Ref. 1). The solid line is a guide to the eye while the dashed line connects the spin-polarized results of Aldén et al. (Ref. 47). 


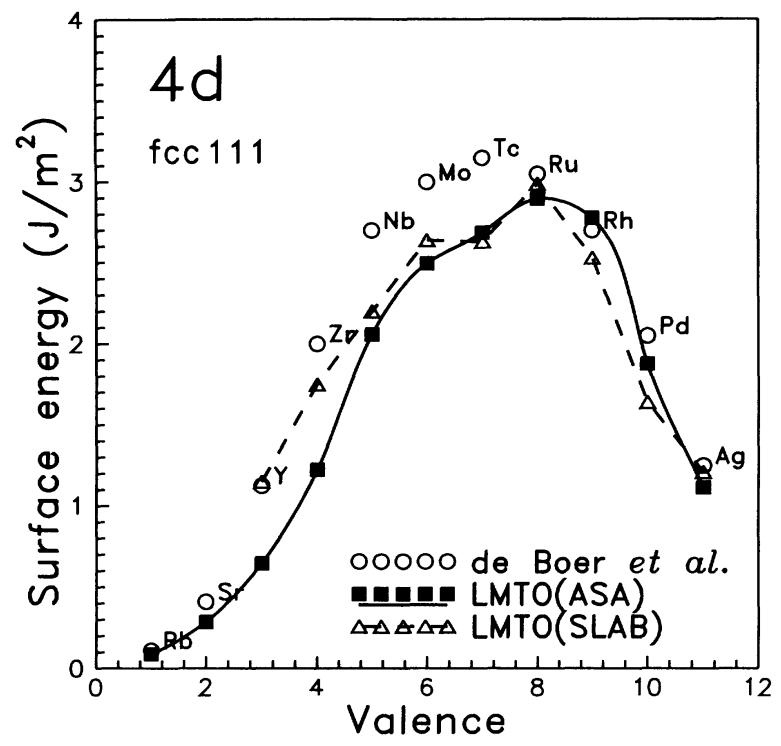

FIG. 5. The calculated surface energy for the fcc (111) surfaces of the $4 d$ metals, solid squares, compared to the structure-independent surface energies derived from the surface tension of the liquid metals, open circles (Ref. 1). The solid line is a guide to the eye while the dashed line connects the full-potential, all-electron, slab-supercell results of Methfessel, Hennig, and Scheffler (Ref. 30).

of surface properties of a transition-metal series by means of a traditional one-electron technique. Within the slabsupercell approximation their full-potential, all-electron method is expected to yield highly accurate surface energies and work functions, and we have therefore included their results in Table III for comparison.

\section{Surface energy}

The calculated surface energies of the fcc (111) surfaces of the transition metals shown in Figs. 4-6 exhibit a parabolic variation with the valence, defined as the total number of $s, p$, and $d$ electrons. This is in accord with the simple picture of $d$-electron contribution to the surface energy suggested by Friedel. ${ }^{46}$ Assuming a constant state density with a bandwidth for $d$ electrons which is reduced by $\delta W$ at the surface, one finds the $d$-electron contribution to the surface energy to be given by

$$
E_{S}=\frac{1}{2} n(1-n / 10) \delta W
$$

where $n$ is the number of $d$ electrons. If we disregard the anomaly in the $3 d$ series, which was recently explained in terms of magnetic effects by Aldén et al. ${ }^{47}$ the parabolic behavior is also obeyed by the surface-independent surface energies derived from the surface tension of the liquid metals. ${ }^{1}$ Hence, the trends exhibited by the experimentally derived surface energies of the $3 d, 4 d$, and $5 d$ transition metals are completely described by the present type of calculation.

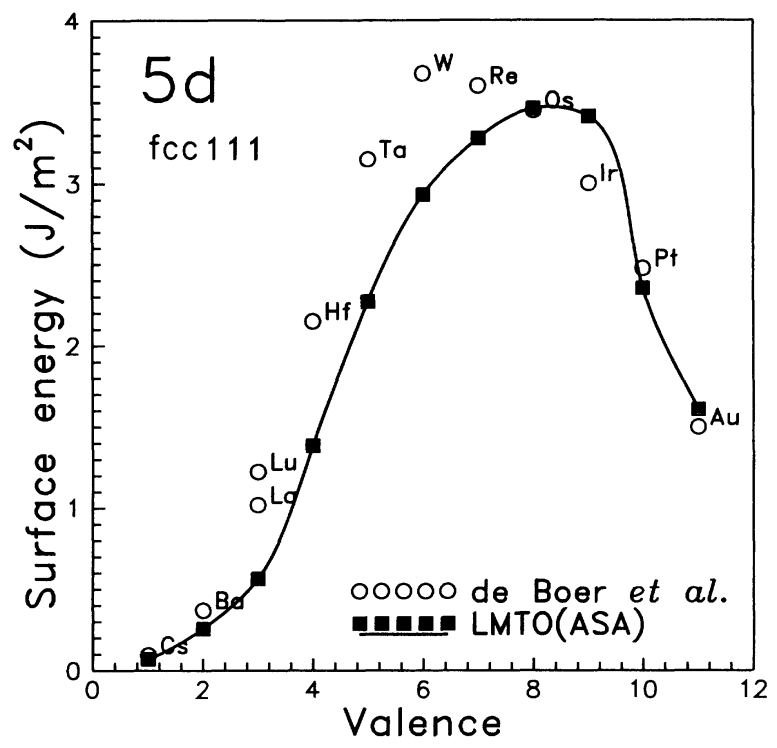

FIG. 6. The calculated surface energy for the fcc (111) surfaces of the $5 d$ metals, solid squares, compared to the structure-independent surface energies derived from the surface tension of the liquid metals, open circles (Ref. 1). The solid line is a guide to the eye.

Examination of Figs. 4-6 and Tables II-IV reveals that the calculated surface energies for the heavy elements in each transition series are in close agreement with experiment, while for the lighter elements they tend to be lower than the experimental values. In view of the approximations used in the reduction of the surface-tension data to zero temperature, this agreement is surprisingly good and lends credibility to the calculated as well as the experimentally derived surface energies.

To judge the accuracy of our LMTO-ASA Green'sfunction approach we have included the fcc (111) surface energies obtained by Methfessel, Hennig, and Scheffler ${ }^{30}$ in Fig. 5. For the first metals in the $4 d$ series, $\mathrm{Y}$ and $\mathrm{Zr}$, the difference between the two calculations is $43 \%$ and $30 \%$, respectively. Part of this large discrepancy may be due to the fact that the present calculations are performed at the experimentally observed equilibrium volumes while those by Methfessel, Hennig, and Scheffler ${ }^{30}$ are performed at the somewhat lower calculated equilibrium volumes. For the heavier metals, $\mathrm{Nb}-\mathrm{Ag}$, on the other hand the agreement between the two sets of calculations is seen to be better than $10 \%$ which we find extremely satisfactory in view of the fact that the methods applied differ in both approach and level of approximation.

\section{Work function}

The calculated work functions of the transition-metal series including the alkali, alkaline earth, and noble metals, are presented in Figs. 7-9 together with the experimental values compiled by Michaelson, ${ }^{2}$ supplemented 
TABLE II. Work functions and surface energies for the $3 d$ metals. Values for ferromagnetic Fe, $\mathrm{Co}$, and $\mathrm{Ni}$ are taken from Aldén et al. (Ref. 42) and denoted by an $F$ after the surface label. The experimental work functions in parentheses are polycrystalline values.

\begin{tabular}{|c|c|c|c|c|c|c|}
\hline \multirow[t]{3}{*}{$\overline{\text { Metal }}$} & \multirow[t]{3}{*}{ Surface } & \multicolumn{2}{|c|}{ Work function } & \multicolumn{3}{|c|}{ Surface energy } \\
\hline & & \multirow{2}{*}{$\begin{array}{c}\text { Theory } \\
(\mathrm{eV})\end{array}$} & \multirow{2}{*}{$\begin{array}{c}\text { Expt. }^{a} \\
(\mathrm{eV})\end{array}$} & \multicolumn{2}{|c|}{ Theory } & \multirow{2}{*}{$\begin{array}{l}\text { Expt. }^{b} \\
\left(\mathrm{~J} / \mathrm{m}^{2}\right)\end{array}$} \\
\hline & & & & $(\mathrm{eV})$ & $\left(\mathrm{J} / \mathrm{m}^{2}\right)$ & \\
\hline \multirow[t]{2}{*}{$\mathrm{Sc}$} & hcp (001) & 3.74 & $(3.5)$ & 0.48 & 0.82 & 1.28 \\
\hline & fcc $(111)$ & 3.84 & & 0.44 & 0.76 & \\
\hline \multirow[t]{2}{*}{$\mathrm{Ti}$} & hcp (001) & 4.59 & $(4.33)$ & 0.90 & 1.95 & 2.10 \\
\hline & fcc $(111)$ & 4.63 & & 0.72 & 1.56 & \\
\hline \multirow[t]{2}{*}{$\mathrm{V}$} & bcc (110) & 5.12 & $(4.3)$ & 0.82 & 2.02 & 2.55 \\
\hline & fcc $(111)$ & 4.88 & & 0.99 & 2.55 & \\
\hline \multirow[t]{2}{*}{$\mathrm{Cr}$} & bcc (110) & 5.45 & $(4.5)$ & 1.33 & 3.63 & 2.30 \\
\hline & fcc (111) & 5.27 & & 1.10 & 3.09 & \\
\hline $\mathrm{Mn}$ & fcc (111) & 5.45 & $(4.1)$ & 1.17 & 3.24 & 1.60 \\
\hline \multirow[t]{3}{*}{$\mathrm{Fe}$} & $\mathrm{bcc}(110) F^{\mathrm{c}}$ & 5.16 & $(4.5)$ & 0.96 & 2.66 & 2.48 \\
\hline & bcc $(110)$ & 5.78 & & 1.12 & 3.09 & 2.48 \\
\hline & fcc $(111)$ & 5.54 & & 1.15 & 3.28 & \\
\hline \multirow[t]{3}{*}{ Co } & hcp $(001) F^{c}$ & 5.48 & $(5.0)$ & 0.94 & 2.74 & 2.55 \\
\hline & hcp $(001)$ & 5.81 & & 1.08 & 3.18 & 2.55 \\
\hline & fcc $(111)$ & 5.76 & & 1.10 & 3.23 & \\
\hline \multirow[t]{2}{*}{$\mathrm{Ni}$} & fcc $(111) F^{c}$ & 5.68 & 5.35 & 0.90 & 2.69 & 2.45 \\
\hline & fcc $(111)$ & 5.77 & 5.35 & 0.88 & 2.63 & 2.45 \\
\hline \multirow[t]{3}{*}{$\mathrm{Cu}$} & fcc (111) & 5.30 & 4.94 & 0.69 & 1.96 & 1.83 \\
\hline & fcc $(100)$ & 5.26 & 4.59 & 0.85 & 2.09 & \\
\hline & fcc $(110)$ & 4.48 & 4.48 & 1.33 & 2.31 & \\
\hline
\end{tabular}

${ }^{a}$ See Ref. 2.

${ }^{\mathrm{b}}$ See Ref. 1.

${ }^{\mathrm{c}}$ See Ref. 42.

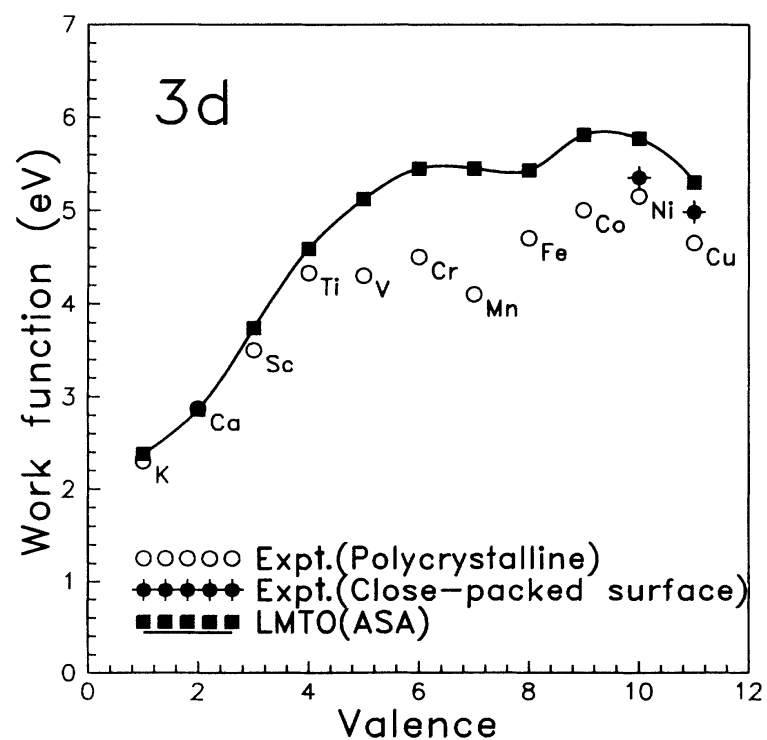

FIG. 7. The calculated work function for the $3 d$ series including the alkali, alkaline earth, and noble metals compared to available experimental polycrystalline data, open circles, and single-surface data, solid circles (Ref. 2). All calculations are performed at the most close-packed surface of the experimentally observed crystal structure. The solid line connecting the theoretical values is a guide to the eye.

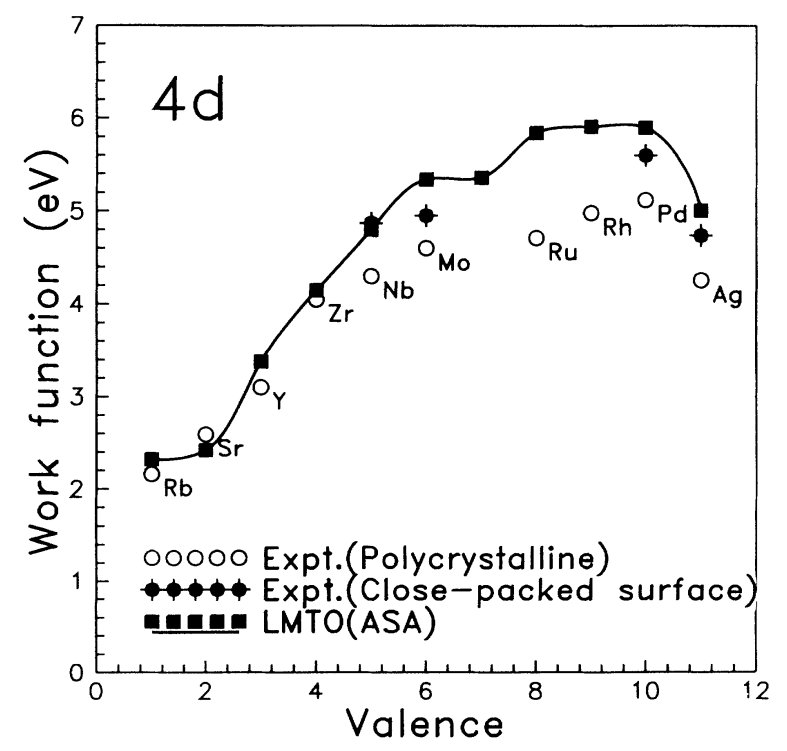

FIG. 8. The calculated work function for the $4 d$ series including the alkali, alkaline earth, and noble metals compared to available experimental polycystalline data, open circles, and single-surface data, solid circles (Ref. 2). All calculations are performed at the most close-packed surface of the experimentally observed crystal structure. The solid line connecting the theoretical values is a guide to the eye. 
TABLE III. Work functions and surface energies for the $4 d$ metals. The experimental work functions in parentheses are polycrystalline values.

\begin{tabular}{|c|c|c|c|c|c|c|c|c|}
\hline \multirow[t]{3}{*}{ Metal } & \multirow[t]{3}{*}{ Surface } & \multicolumn{3}{|c|}{ Work function } & \multicolumn{3}{|c|}{ Surface energy } & \multirow{3}{*}{$\begin{array}{l}\text { Expt. }^{c} \\
\left(\mathrm{~J} / \mathrm{m}^{2}\right)\end{array}$} \\
\hline & & \multirow{2}{*}{$\begin{array}{c}\text { Slab }^{a} \\
(\mathrm{eV})\end{array}$} & \multirow{2}{*}{$\begin{array}{c}\text { Present } \\
(\mathrm{eV})\end{array}$} & \multirow{2}{*}{$\begin{array}{c}\text { Expt. }^{\mathrm{b}} \\
(\mathrm{eV})\end{array}$} & \multirow{2}{*}{$\begin{array}{l}\mathrm{Slab}^{\mathrm{a}} \\
\left(\mathrm{J} / \mathrm{m}^{2}\right)\end{array}$} & \multicolumn{2}{|c|}{ Present } & \\
\hline & & & & & & $(\mathrm{eV})$ & $\left(\mathrm{J} / \mathrm{m}^{2}\right)$ & \\
\hline \multirow[t]{2}{*}{$\mathrm{Y}$} & hcp (001) & & 3.38 & $(3.1)$ & & 0.48 & 0.68 & 1.13 \\
\hline & fcc $(111)$ & 3.46 & 3.42 & & 1.15 & 0.45 & 0.65 & \\
\hline \multirow[t]{2}{*}{$\mathrm{Zr}$} & hcp (001) & & 4.15 & $(4.05)$ & & 0.85 & 1.53 & 2.00 \\
\hline & fcc (111) & 4.38 & 4.35 & & 1.75 & 0.68 & 1.22 & \\
\hline \multirow[t]{2}{*}{$\mathrm{Nb}$} & bcc (110) & 4.66 & 4.80 & 4.87 & 2.36 & 0.79 & 1.64 & 2.70 \\
\hline & fcc $(111)$ & 4.63 & 4.69 & (4.3) & 2.20 & 0.96 & 2.06 & \\
\hline \multirow[t]{2}{*}{ Mo } & bcc (110) & 4.94 & 5.34 & 4.95 & 3.14 & 1.38 & 3.18 & 3.00 \\
\hline & fcc (111) & 4.98 & 5.09 & $(4.6)$ & 2.64 & 1.06 & 2.50 & \\
\hline \multirow[t]{2}{*}{$\mathrm{Tc}$} & hcp (001) & & 5.36 & & & 1.12 & 2.80 & 3.15 \\
\hline & fcc (111) & 5.15 & 5.42 & & 2.63 & 1.06 & 2.69 & \\
\hline \multirow[t]{2}{*}{$\mathrm{Ru}$} & hcp (001) & & 5.84 & $(4.71)$ & & 1.28 & 3.32 & 3.05 \\
\hline & fcc $(111)$ & 5.33 & 5.73 & & 2.99 & 1.12 & 2.90 & \\
\hline \multirow[t]{2}{*}{$\mathrm{Rh}$} & fcc (111) & 5.44 & 5.91 & $(4.98)$ & 2.53 & 1.09 & 2.78 & 2.70 \\
\hline & fcc $(100)$ & 5.25 & 6.14 & & 2.81 & 1.31 & 2.90 & \\
\hline \multirow[t]{2}{*}{$\mathrm{Pd}$} & fcc (111) & 5.53 & 5.90 & 5.6 & 1.64 & 0.77 & 1.88 & 2.05 \\
\hline & fcc $(100)$ & 5.30 & 5.96 & & 1.86 & 0.90 & 1.90 & \\
\hline \multirow[t]{3}{*}{$\mathrm{Ag}$} & fcc (111) & 4.67 & 5.01 & 4.74 & 1.21 & 0.50 & 1.12 & 1.25 \\
\hline & fcc $(100)$ & 4.43 & 5.02 & 4.64 & 1.21 & 0.62 & 1.20 & \\
\hline & fcc $(110)$ & 4.23 & 4.40 & 4.52 & 1.26 & 0.94 & 1.29 & \\
\hline
\end{tabular}

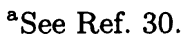

${ }^{\mathrm{b}}$ See Ref. 2.

${ }^{\mathrm{c}}$ See Ref. 1.

by recent results for $\mathrm{Pt}^{48}$ In the comparison with experiment we find that the calculated work function is in good agreement with the single-crystal data and generally above the corresponding polycrystalline data. This is in agreement with the fact that the calculations are performed for the most close-packed surfaces which have the highest work functions, and hence the theory gives a correct description of the trends exhibited by the experimentally observed work function for the $3 d, 4 d$, and $5 d$ metals.

In most of the cases where single-surface data exist, the agreement between theory and experiment is particularly good. This applies to $\mathrm{Ni}, \mathrm{Cu}, \mathrm{Pd}$, and $\mathrm{Ag}$ fcc (111) and to $\mathrm{V}, \mathrm{Nb}, \mathrm{Mo}, \mathrm{Ta}$, and $\mathrm{W}$ bcc (110) where the differences range from 0.0 to $0.4 \mathrm{eV}$. For Re the difference is also small, but here the measurement is performed on the hcp (1011) surface, and hence should not be compared directly with the theoretical hcp (001) value. The largest discrepancies in terms of absolute values are found at the end of the $5 d$ series, where the differencies for $\mathrm{Ir}, \mathrm{Pt}$, and $\mathrm{Au}$ fcc (111) range from 0.7 to $0.9 \mathrm{eV}$, which, however, is only of the order of $15 \%$.

To judge the accuracy of the present approach we compare in Fig. 10 the work functions for the fcc (111) surfaces of the $4 d$ metals as obtained by our LMTO-ASA Green's-function technique with those obtained by the full-potential, all-electron, slab-supercell calculations of

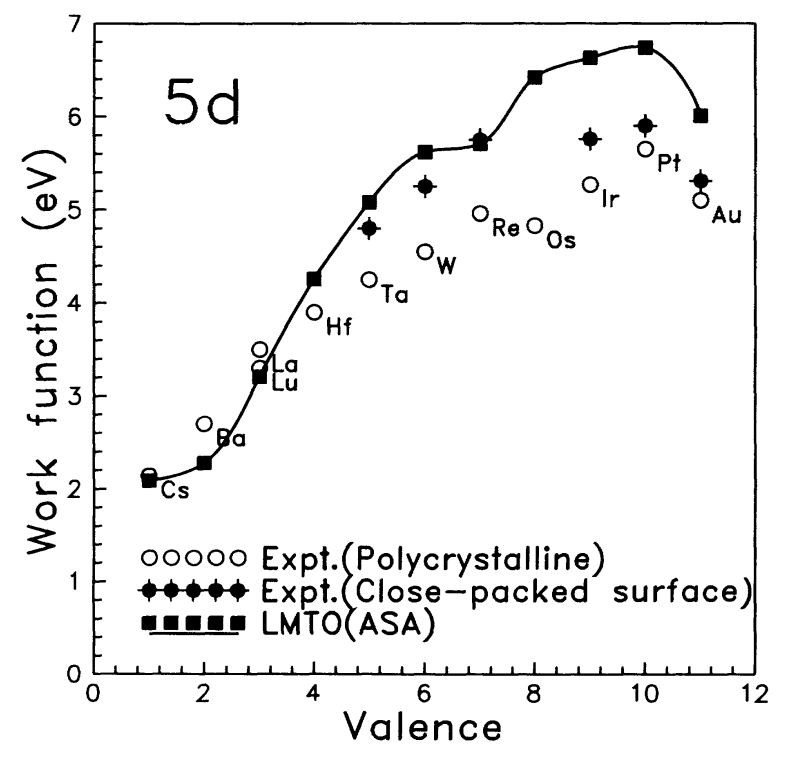

FIG. 9. The calculated work function for the $5 d$ series including the alkali, alkaline earth, and noble metals compared to available experimental polycrystalline data, open circles, and single-surface data, solid circles (Refs. 2 and 48). All calculations are performed at the most close-packed surface of the experimentally observed crystal structure. The solid line connecting the theoretical values is a guide to the eye. 
TABLE IV. Work functions and surface energies for the $5 d$ metals. The experimental work functions in parentheses are polycrystalline values.

\begin{tabular}{|c|c|c|c|c|c|c|}
\hline \multirow[t]{3}{*}{ Metal } & \multirow[t]{3}{*}{ Surface } & \multicolumn{2}{|c|}{ Work function } & \multicolumn{3}{|c|}{ Surface energy } \\
\hline & & \multirow{2}{*}{$\begin{array}{c}\text { Theory } \\
(\mathrm{eV})\end{array}$} & \multirow{2}{*}{$\begin{array}{c}\text { Expt. }^{a} \\
(\mathrm{eV})\end{array}$} & \multicolumn{2}{|c|}{ Theory } & \multirow{2}{*}{$\begin{array}{l}\text { Expt. } \\
\left(\mathrm{J} / \mathrm{m}^{2}\right)\end{array}$} \\
\hline & & & & $(\mathrm{eV})$ & $\left(\mathrm{J} / \mathrm{m}^{2}\right)$ & \\
\hline \multirow[t]{2}{*}{$\mathrm{La}$} & hcp (001) & 3.21 & $(3.5)$ & 0.43 & 0.57 & 1.02 \\
\hline & fcc $(111)$ & 3.30 & & 0.43 & 0.57 & \\
\hline \multirow[t]{2}{*}{$\mathrm{Lu}$} & hcp (001) & 3.57 & $(3.3)$ & 0.50 & 0.77 & 1.23 \\
\hline & fcc $(111)$ & 3.65 & & 0.47 & 0.72 & \\
\hline \multirow[t]{2}{*}{$\mathrm{Hf}$} & hcp (001) & 4.26 & $(3.9)$ & 0.94 & 1.75 & 2.15 \\
\hline & fcc $(111)$ & 4.54 & & 0.75 & 1.39 & \\
\hline \multirow[t]{2}{*}{$\mathrm{Ta}$} & bcc (110) & 5.08 & 4.80 & 0.86 & 1.79 & 3.15 \\
\hline & fcc $(111)$ & 4.86 & $(4.25)$ & 1.06 & 2.27 & \\
\hline \multirow[t]{2}{*}{$\mathrm{W}$} & bcc (110) & 5.62 & 5.25 & 1.70 & 3.84 & 3.68 \\
\hline & fcc $(111)$ & 5.09 & $(4.55)$ & 1.06 & 2.50 & \\
\hline \multirow[t]{2}{*}{$\mathrm{Re}$} & hcp (001) & 5.71 & $5.75^{c}$ & 1.34 & 3.27 & 3.60 \\
\hline & fcc $(111)$ & 5.88 & & 1.34 & 3.28 & \\
\hline \multirow[t]{2}{*}{ Os } & hcp (001) & 6.42 & $(4.83)$ & 1.60 & 4.04 & 3.45 \\
\hline & fcc $(111)$ & 6.25 & & 1.37 & 3.46 & \\
\hline \multirow[t]{2}{*}{$\mathrm{Ir}$} & fcc $(111)$ & 6.63 & 5.76 & 1.36 & 3.41 & 3.00 \\
\hline & fcc $(100)$ & 7.05 & 5.67 & 1.75 & 3.81 & \\
\hline \multirow[t]{2}{*}{$\mathrm{Pt}$} & fcc $(111)$ & 6.74 & $5.7^{\mathrm{d}}$ & 0.98 & 2.35 & 2.48 \\
\hline & fcc $(100)$ & 6.97 & & 1.19 & 2.48 & \\
\hline \multirow[t]{3}{*}{$\mathrm{Au}$} & fcc (111) & 6.01 & 5.31 & 0.72 & 1.61 & 1.50 \\
\hline & fcc $(100)$ & 6.16 & 5.47 & 0.88 & 1.71 & \\
\hline & fcc (110) & 5.40 & 5.40 & 1.31 & 1.79 & \\
\hline
\end{tabular}

${ }^{\text {a See Ref. } 2 .}$

bee Ref. 1.

${ }^{c}$ hcp (1011).

${ }^{\mathrm{d}}$ See Ref. 48.

Methfessel, Hennig, and Scheffler. ${ }^{30}$ It is seen that for the entire series, $\mathrm{Y}-\mathrm{Ag}$, the agreement between the two sets of calculations is better than $9 \%$ which again is extremely satisfactory.

It is perhaps surprising that the work function varies so smoothly through a transition series. To understand this, we may consider the two terms in Eq. (22) separately, and since the work functions of the three transition series follow the same trend, it will suffice to explain the variation exhibited by the $4 d$ series on the basis of the calculated Fermi level and dipole barrier shown in Fig. 11.

Pettifor, ${ }^{49}$ Mackintosh and Andersen, ${ }^{50}$ and Glötzel ${ }^{51}$ found in agreement with our bulk results that the Fermi level in the $4 d$ series exhibits a maximum close to Mo. They explained this behavior as the effect of a repulsive ion core whose size, relative to the atomic sphere, attains a maximum in the middle of the series. The $s$ electrons respond to being confined in the narrow volume between the core and the atomic sphere by increasing the mean band energy and the bandwidth. Since the number of non- $d$ states remains essentially independent of atomic number, the "parabolic" behavior of the Fermi level follows.

In a metal, the relative confinement of the $s$ electrons

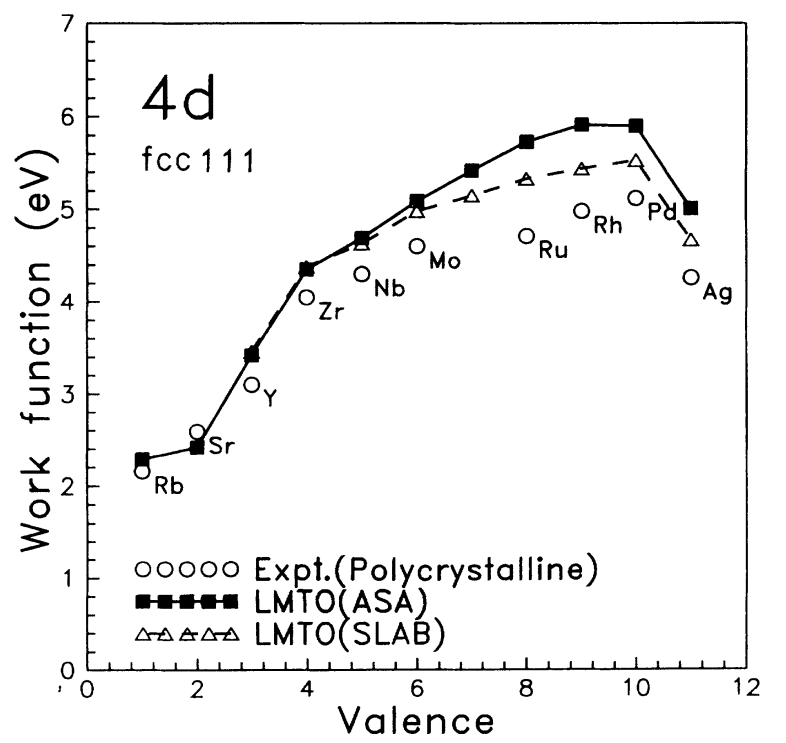

FIG. 10. The calculated work functions for the fcc (111) surfaces of the $4 d$ metals, solid squares, compared to the available experimental polycrystalline data, open circles (Ref. 2). The solid line is a guide to the eye while the dashed line connects the full-potential, all-electron, slab-supercell results of Methfessel, Hennig, and Scheffler (Ref. 30). 


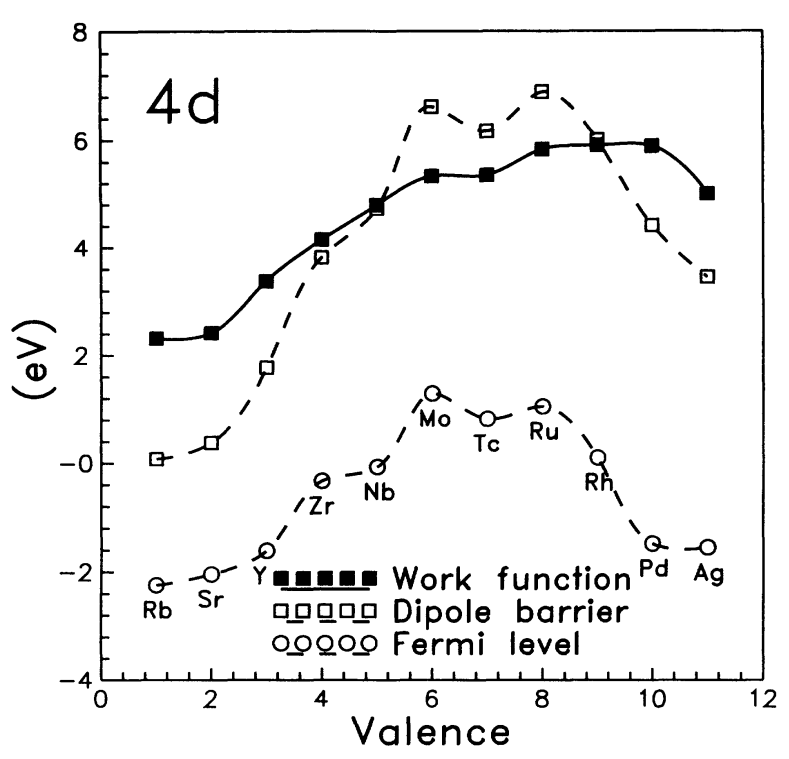

FIG. 11. The calculated work function, dipole barrier, and Fermi level for the $4 d$ metals as a function of the total number of $s, p$, and $d$ electrons .

results in an increase in the kinetic energy, and for that reason the bulk modulus attains a maximum close to the middle of a transition series. At a surface the $s$ electrons will reduce their kinetic energy by spilling out into the vacuum, and therefore the surface dipole also exhibits a "parabolic" dependence on atomic number. As a consequence, the work function, obtained as the difference Eq. (22), varies smoothly with atomic number, in agreement with experiment.

Nieminen and Hodges ${ }^{8}$ reversed Eq. (22) and deduced the surface dipole barrier for transition metals from band estimates of the Fermi level and the experimentally obtained work function. Their results agree with our firstprinciples values to within $10 \%-30 \%$ which may be considered a surprisingly good agreement in view of the large uncertainties in their procedure. Thus, the present calculations substantiate the notion ${ }^{8}$ that the width of the dipole layer varies little with atomic number, because it is governed by the screening length, and that the height of the dipole barrier therefore to a large degree is determined by the charge which is able to reduce its kinetic energy by flowing out into the vacuum.

\section{Anisotropy}

The surface energy and the work function of a given metal depend on the crystallographic orientation, i.e., the surface facet, of the surface under consideration. If one assumes that the surface energy is proportional to the number of bonds being cut in the formation of the surface, one finds for an fcc crystal that

$$
\gamma_{(110)}>\gamma_{(100)}>\gamma_{(111)}
$$

which are also the inequalities found in tight-binding studies. ${ }^{52,53}$ The anisotropy of the work function is determined by the electrostatic dipole barrier (23), and based on geometric considerations alone one finds the inequalities

$$
W_{(111)}>W_{(100)}>W_{(110)}
$$

In general, one expects the surface energy to increase and the work function to decrease as the surface becomes more open.

Examination of Tables I-IV shows that the present surface energies for the alkali metals, $\mathrm{Li}$ and $\mathrm{Na}$, in contrast to the jellium results, do not follow the order $\gamma_{(100)}>\gamma_{(110)}$ expected for bcc crystals. However, for the heavier alkali metals, $\mathrm{K}-\mathrm{Cs}$, and for the noble metals the order is the same as that given by the broken-bond model (26). Similarly, the present work functions of the alkali metals in Table I exhibit the order expexted for bcc surfaces, in complete agreement with the jellium results. The expected order (27) is also found for the work functions of $\mathrm{Cu}$, Table II, in agreement with experiment but for Ag, Table III, the work function of the fcc (100) surface is similar to that of the fcc (111) surface, in contrast to both slab calculations and experiment. For $\mathrm{Au}$ the order does not follow the simple model but here the calculation is in agreement with experiment.

\section{DISCUSSION}

The determination of the surface energy and the work function of a metal is of great importance in the understanding of a wide range of surface phenomena, including the growth rate, the form of crystallites, sintering, catalytic behavior, adsorption, surface segregation, and the formation of grain boundaries. Traditionally these properties have been estimated from a variety of surface measurements using procedures which bring about uncertainties in the recommended values of unknown magnitude.

For instance, de Boer et al. ${ }^{1}$ have estimated the surface energy of a metal by subtracting from the measured surface tension of the liquid an entropy term proportional to the melting temperature. Although this may seem a reasonable procedure, it can only be justified by the validity of the physical picture it leads to, or by the degree to which it agrees with $a b$ initio calculations. The fact that two very different sets of calculations are in closer agreement with each other than they individually agree with experiment (see Fig. 5) strongly suggest that present day $a b$ initio calculations may be the most accurate way of estimating surface energies.

If one considers the difference between the work function measured on a polycrystalline sample and the work function measured on a single crystal of the same metal as a measure of the uncertainty in the experimentally derived work functions, one finds that the values recommended by Michaelson ${ }^{2}$ are in error by up to $1 \mathrm{eV}$. Again, this uncertainty is larger than the difference between present day $a b$ initio calculations (see Fig. 10) and 
suggests that such calculations may form a most valuable tool for estimating work functions.

Based on the present experience, we would argue that for properties as difficult to assess experimentally as surface properties, $a b$ initio calculations have reached a stage where they may form the most consistent basis for a physical description of surface phenomena.

\section{ACKNOWLEDGMENTS}

This work was supported by grants from the Novo Foundation, the Danish Natural Science Foundation (SNF), and the Danish Technical Science Foundation (STVF) under the development program for materials (MUP).
${ }^{1}$ F. R. de Boer, R. Boom, W. C. M. Mattens, A. R. Miedema, and A. K. Niessen, Cohesion in Metals (North-Holland, Amsterdam, 1988).

${ }^{2}$ H. B. Michaelson, J. Appl. Phys. 48, 4729 (1977).

${ }^{3}$ N. D. Lang and W. Kohn, Phys. Rev. B 1, 4555 (1970).

${ }^{4}$ N. D. Lang and W. Kohn, Phys. Rev. B 3, 1215 (1971).

${ }^{5}$ R. Monnier and J. P. Perdew, Phys. Rev. B 17, 2595 (1978); 22, 1124(E) (1980).

${ }^{6}$ Z. Y. Zhang, D. C. Langreth, and J. P. Perdew, Phys. Rev. B 41, 5674 (1990).

${ }^{7}$ J. P. Perdew, H. Q. Tran, and E. D. Smith, Phys. Rev. B 42, 11627 (1990).

${ }^{8}$ R. M. Nieminen and C. H. Hodges, J. Phys. F 6, 573 (1976).

${ }^{9}$ M. Weinert and R. E. Watson, Phys. Rev. B 29, 3001 (1984).

${ }^{10} \mathrm{O}$. Jepsen, J. Madsen, and O. K. Andersen, Phys. Rev. B 18, 605 (1978).

${ }^{11}$ J. A. Appelbaum and D. R. Hamann, Solid State Commun. 27, 881 (1978).

${ }^{12}$ M. Posternak, H. Krakauer, A. J. Freeman, and D. Koelling, Phys. Rev. B 21, 5601 (1980).

${ }^{13}$ J. R. Smith, J. G. Gay, and F. J. Arlinghaus, Phys. Rev. B 21, 2201 (1980); F. J. Arlinghaus, J. G. Gay, and J. R. Smith, ibid. 23, 5152 (1981).

${ }^{14}$ P. J. Feibelman, Phys. Rev. B 26, 5347 (1982).

${ }^{15}$ O. Jepsen, J. Madsen, and O. K. Andersen, Phys. Rev. B 26, 2790 (1982).

${ }^{16}$ D.-S. Wang, A. J. Freeman, and H. Krakauer, Phys. Rev. B 26, 1340 (1982).

${ }^{17}$ A. Euceda, D. M. Bylander, L. Kleinman, and K. Mednick, Phys. Rev. B 27, 659 (1983).

${ }^{18} \mathrm{~S}$. Ohnishi, A. J. Freeman, M. Wienert, and K. Mednick, Phys. Rev. B 28, 6741 (1983).

${ }^{19}$ H. Krakauer, Phys. Rev. B 30, 6834 (1984).

${ }^{20}$ L. F. Mattheiss and D. R. Hamann, Phys. Rev. B 29, 5372 (1984).

${ }^{21}$ G. W. Fernando, B. R. Cooper, M. V. Ramana, H. Krakauer, and C. Q. Ma, Phys. Rev. Lett. 56, 2299 (1986).

${ }^{22}$ G. W. Fernando and J. W. Wilkins, Phys. Rev. B 33, 3709 (1986).

${ }^{23}$ O. Jepsen and R. O. Jones, Phys. Rev. B 34, 6695 (1986).

${ }^{24}$ K.-M. Ho and K. P. Bohnen, Phys. Rev. Lett. 59, 1833 (1987).

${ }^{25}$ J. E. Inglesfield and G. A. Benesh, Phys. Rev. B 37, 6682 (1988)

${ }^{26}$ R. J. Needs and M. Mansfield, J. Phys. Condens. Matter 1, 7555 (1989).

${ }^{27}$ H. Erschbaumer, A. J. Freeman, C. L. Fu, and R. Pod- loucky, Surf. Sci. 243, 317 (1991).

${ }^{28}$ H. L. Skriver and N. M. Rosengaard, Phys. Rev. B 43, 9538 (1991).

${ }^{29}$ H. L. Skriver and N. M. Rosengaard, Phys. Rev. B 45, 9410 (1992).

${ }^{30}$ M. Methfessel, D. Hennig, and M. Scheffler, Phys. Rev. B (to be published).

${ }^{31}$ O. K. Andersen, Phys. Rev. B 12, 3060 (1975).

${ }^{32}$ O. Gunnarsson, O. Jepsen, and O. K. Andersen, Phys. Rev. B 27, 7144 (1983).

${ }^{33}$ H. L. Skriver, The LMTO Method (Springer-Verlag, Berlin, 1984).

${ }^{34}$ O. K. Andersen and O. Jepsen, Phys. Rev. Lett. 53, 2571 (1984).

${ }^{35}$ O. K. Andersen, O. Jepsen, and D. Glötzel, in Highlights of Condensed-Matter Theory, edited by F. Bassani, F. Fumi, and M. P. Tosi (North-Holland, New York, 1985).

${ }^{36}$ O. K. Andersen, Z. Pawlowska, and O. Jepsen, Phys. Rev. B 34, 5253 (1986).

${ }^{37}$ W. Lambrecht and O. K. Andersen, Surf. Sci. 178, 256 (1986); (private communication).

${ }^{38}$ P. O. Löwdin, J. Chem. Phys. 19, 1396 (1951).

${ }^{39}$ W. Lambrecht, B. Segall, and O. K. Andersen, Phys. Rev. B 41, 2813 (1990).

${ }^{40}$ S. L. Cunningham, Phys. Rev. 10, 4988 (1974).

${ }^{41}$ R. Zeller, P. Lang, B. Drittler, and P. H. Dederichs (unpublished).

${ }^{42}$ M. E. Aldén, S. Mirbt, H. L. Skriver, N. M. Rosengaard, and B. Johansson, Phys. Rev. B 46, 6303 (1992).

${ }^{43}$ D. M. Ceperley and B. J. Alder, Phys. Rev. Lett. 45, 566 (1980).

${ }^{44}$ J. Perdew and A. Zunger, Phys. Rev. B 23, 5048 (1981).

${ }^{45} \mathrm{See}$, for instance, Fig. 2.2 of Ref. 1.

${ }^{46}$ J. Friedel, Ann. Phys. (N.Y.) 1, 257 (1976).

${ }^{47}$ M. E. Aldén, H. L. Skriver, S. Mirbt, and B. Johansson (unpublished).

${ }^{48}$ M. Kiskinova, G. Pirug, and H. P. Bonzel, Surf. Sci. 133, 321 (1983).

${ }^{49}$ D. G. Pettifor, J. Phys. F 7, 613 (1977).

${ }^{50} \mathrm{~A}$. R. Mackintosh and O. K. Andersen, in Electrons at the Fermi Surface, edited by M. Springford (Cambridge University Press, Cambridge, 1980), p. 149.

${ }^{51}$ D. Glötzel, in Physics of Solids under High Pressure, edited by J. S. Shilling and R. N. Shelton (North-Holland, Amsterdam, 1981), p. 263.

${ }^{52}$ F. Cyrot-Lackmann, Surf. Sci. 15, 535 (1969).

${ }^{53} \mathrm{M}$. C. Desjonqueres and F. Cyrot-Lackmann, Surf. Sci. 50, 257 (1975). 